\title{
HIF-independent regulation of VEGF and angiogenesis by the transcriptional coactivator PGC-1a
}

\author{
Zoltan Arany ${ }^{1,2}$, Shi-Yin Foo ${ }^{2}$, Yanhong Ma ${ }^{1}$, Jorge L. Ruas ${ }^{1}$, Archana Bommi-Reddy ${ }^{1}$, Geoffrey Girnun ${ }^{1}$, \\ Marcus Cooper ${ }^{1}$, Dina Laznik ${ }^{1}$, Jessica Chinsomboon ${ }^{1}$, Shamina M. Rangwala ${ }^{3}$, Kwan Hyuck Baek ${ }^{4}$, \\ Anthony Rosenzweig ${ }^{2} \&$ Bruce M. Spiegelman ${ }^{1}$
}

Ischaemia of the heart, brain and limbs is a leading cause of morbidity and mortality worldwide. Hypoxia stimulates the secretion of vascular endothelial growth factor (VEGF) and other angiogenic factors, leading to neovascularization and protection against ischaemic injury ${ }^{1}$. Here we show that the transcriptional coactivator PGC-1 $\alpha$ (peroxisome-proliferator-activated receptor- $\gamma$ coactivator$1 \alpha$ ), a potent metabolic sensor and regulator ${ }^{2}$, is induced by a lack of nutrients and oxygen, and PGC-1 $\alpha$ powerfully regulates VEGF expression and angiogenesis in cultured muscle cells and skeletal muscle in vivo. $P G C-1 \alpha^{-1-}$ mice show a striking failure to reconstitute blood flow in a normal manner to the limb after an ischaemic insult, whereas transgenic expression of PGC-1 $\alpha$ in skeletal muscle is protective. Surprisingly, the induction of VEGF by PGC-1 $\alpha$ does not involve the canonical hypoxia response pathway and hypoxia inducible factor (HIF). Instead, PGC-1 $\alpha$ coactivates the orphan

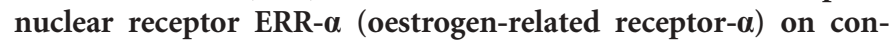
served binding sites found in the promoter and in a cluster within the first intron of the VEGF gene. Thus, PGC- $1 \alpha$ and ERR- $\alpha$, major regulators of mitochondrial function in response to exercise and other stimuli, also control a novel angiogenic pathway that delivers needed oxygen and substrates. PGC-1 $\alpha$ may provide a novel therapeutic target for treating ischaemic diseases.

Ischaemia is a profound metabolic challenge with potentially catastrophic consequences. PGC- $1 \alpha$ is a potent modulator of oxidative metabolism in numerous settings ${ }^{2}$. In particular, PGC- $1 \alpha$ powerfully regulates oxidative phosphorylation, mitochondrial biogenesis, and respiration $^{3,4}$. To investigate a possible role for PGC- $1 \alpha$ in ischaemia, cultured $\mathrm{C} 2 \mathrm{C} 12$ myotubes were deprived of nutrients and maintained in $0.2 \%$ oxygen. This led within $3 \mathrm{~h}$ to an induction of more than tenfold in PGC-1 $\alpha$ mRNA, as measured by quantitative PCR (Fig. 1a), and an increase in PGC-1 $\alpha$ protein, as detected by western blotting (Supplementary Fig. 1). The expression of $P G C-1 \alpha$ remained elevated when treatment was continued for $48 \mathrm{~h}$ (Supplementary Fig. 2) and returned to baseline within $3 \mathrm{~h}$ after restoration of complete medium and $21 \%$ oxygen (Fig. 1a). This induction of $P G C-1 \alpha$ was not unique to $\mathrm{C} 2 \mathrm{C} 12$ myotubes and was observed in nearly all cell lines tested, including fibroblasts, striatal neurons, hepatocytes and primary skeletal muscle cells (Supplementary Fig. 3). Either removal of nutrients or placement in $0.2 \%$ oxygen alone led to a threefold induction of $P G C-1 \alpha$ expression, whereas the combined treatment led to a synergistic 12-fold induction (Fig. 1b). Hypoxia is well known to induce the expression of a broad genetic programme, at least in part through stabilization of the transcription factors HIF- $1 \alpha$ and HIF- $2 \alpha$ (ref. 5). This programme includes angiogenic genes such as VEGF and glycolytic genes such as GLUT1. To examine whether a

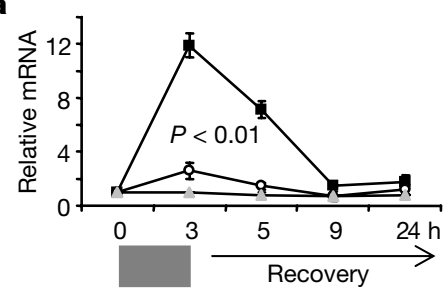

b

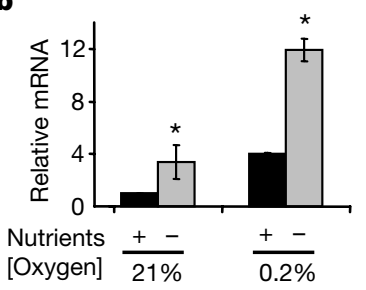

c

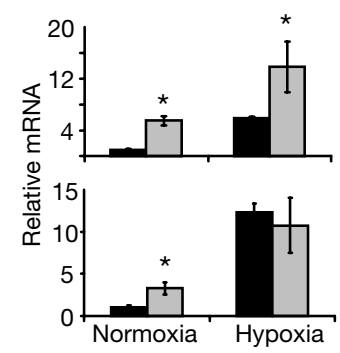

d

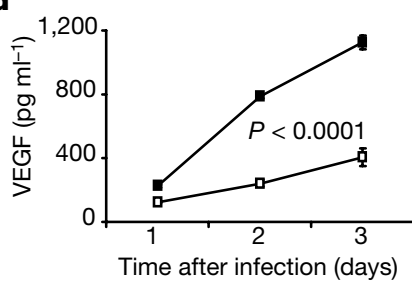

e
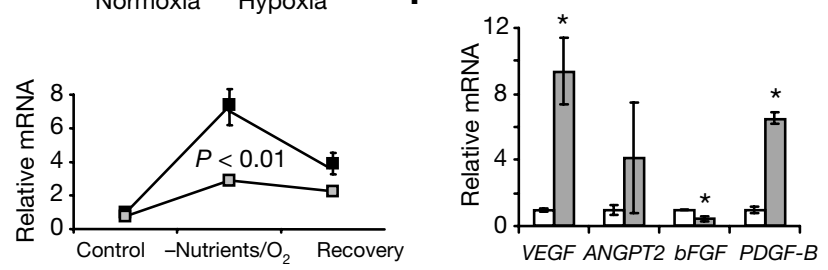

Figure $1 \mid$ PGC-1 $\alpha$ regulates VEGF in response to deprivation of nutrients and oxygen. a, C2C12 myotubes were deprived of oxygen $(0.2 \%)$ and nutrients for $3 \mathrm{~h}$ and then restored to complete medium. Gene expression was measured by quantitative PCR. Squares, $P G C-1 \alpha$; circles, $P G C-1 \beta$; triangles, $P R C$ (PGC-1-related gene). $\mathbf{b}$, Myotubes were deprived of oxygen or nutrients, or both, for $3 \mathrm{~h}$, and expression of $P G C-1 \alpha$ mRNA was measured. c, Myotubes were infected with adenovirus expressing GFP (black bars) or PGC- $1 \alpha$ (grey bars) for $24 \mathrm{~h}$ and then maintained in either $21 \%$ or $0.2 \%$ oxygen for $16 \mathrm{~h}$. Top, VEGF mRNA; bottom, GLUT1 mRNA. d, Myotubes were infected as in c, and VEGF in the culture medium was measured. Open squares, GFP; filled squares, PGC-1 $\alpha$. e, Primary muscle cells derived from $P G C$ - $1 \alpha$ wild-type (black squares) and knockout (grey squares) animals were treated as in a; expression of VEGF mRNA was measured. f, Primary myotubes were infected with adenovirus expressing GFP (white bars) or PGC- $1 \alpha$ (grey bars) and expression of the indicated mRNAs was measured. ANGPT2, angiopoietin-2; bFGF, basic fibroblast growth factor. Error bars indicate s.e.m.; $n>3$ per group in all panels. Asterisk, $P<0.05$ compared with control. 
PGC- $1 \alpha$ contributes to the regulation of this programme, $\mathrm{C} 2 \mathrm{C} 12$ myotubes were infected with adenovirus expressing PGC- $1 \alpha$ or green fluorescent protein (GFP) as control. PGC-1 $\alpha$ expression led to a fourfold induction of VEGF mRNA, which was further increased to 14 -fold in the presence of $0.2 \%$ oxygen (Fig. 1c and Supplementary Fig. 4). The induced VEGF transcript is functional, as demonstrated by an increased secretion of VEGF protein into the cell culture medium (Fig. 1d). All alternatively spliced forms of VEGF were induced by PGC-1 $\alpha$ (Supplementary Fig. 5). To determine whether PGC- $1 \alpha$ is required for the induction of VEGF, primary skeletal muscle cells were acquired from $P G C-1 \alpha^{-1-}$ and wild-type animals. Treating these cells for $3 \mathrm{~h}$ with $0.2 \%$ oxygen and nutrient deprivation led to an 8-fold induction of VEGF in wild-type cells, and this was reduced to threefold in $P G C-1 \alpha^{-/-}$cells (Fig. 1e). The induction of GLUT1 was similarly blunted in $P G C-1 \alpha^{-1-}$ cells (Supplementary Fig. 6). Hence, under these conditions, PGC- $1 \alpha$ contributes greatly to the VEGF gene response to nutrient and oxygen deprivation. Angiogenesis in response to hypoxia or other insults is a complex programme, requiring coordination by multiple signals ${ }^{6}$. VEGF has a dominant function in recruiting the endothelium, whereas plateletderived growth factor (PDGF)-BB recruits mural cells to support and encase the endothelium. Angiopoietin 2, in the presence of VEGF, facilitates the sprouting of new vessels from existing vessels. All three of the genes encoding these proteins are inducible by PGC- $1 \alpha$, as shown by the adenoviral delivery of PGC- $1 \alpha$ to primary skeletal muscle cells (Fig. 1f). Together, these data show that PGC- $1 \alpha$ is markedly induced by nutrient and oxygen deprivation and, in turn, regulates a wide programme of genes involved in the coordination of neovascularization.

We have previously described transgenic animals that express PGC-1 $\alpha$ in some skeletal muscle beds at levels that are about tenfold those in wild-type controls ${ }^{7}$. To test whether PGC- $1 \alpha$ can elicit neovascularization in vivo, we examined the skeletal muscle of these animals, compared with wild-type littermate controls. Expression of VEGF was induced in all muscle beds examined in the transgenic animals (Fig. 2a, top panel), except in the fibre type I-rich soleus muscle; the transgene is less well expressed in soleus and there is little induction of PGC-1 $1 \alpha$ above wild-type levels (data not shown). Expression of other angiogenic factors, including PDGF-B and angiopoietin 2, was also induced in transgenic skeletal muscle (Fig. 2a, bottom panel). The density of capillaries was induced markedly in multiple muscle beds of transgenic animals in comparison with wildtype littermate controls, as determined by staining for the endothelial marker CD31 (Fig. 2b and Supplementary Fig. 7). The number of capillaries per high-power field increased from 100 to 300, and the number of capillaries per fibre similarly increased from five to ten. Hence, PGC-1 $\alpha$ powerfully induces angiogenesis in vivo.

We next tested whether PGC- $1 \alpha$ was required for the normal vascular response of skeletal muscle to ischaemia. $P G C-1 \alpha^{-1-}$ animals and wild-type controls were subjected to ligation and ablation of the femoral artery. In wild-type animals this procedure causes nearly complete cessation of blood flow to the hind limb. This is then followed by progressive neovascularization and return of blood flow to the limb over the ensuing weeks (ref. 8 and Fig. 2c-e). By contrast, the return of blood flow is severely blunted in $P G C-1 \alpha^{-1-}$ animals (Fig. 2c, d); only about $30 \%$ of blood flow is recaptured in $P G C-1 \alpha^{-1-}$ animals 14 days after surgery, in contrast with more than $50 \%$ in wild-type littermate controls. Moreover, in transgenic animals expressing PGC- $1 \alpha$ in skeletal muscle, the return of blood flow after ligation of the femoral artery is markedly accelerated (Fig. 2e and Supplementary Fig. 8): 75\% of blood flow is recaptured in these animals within six days after surgery, in
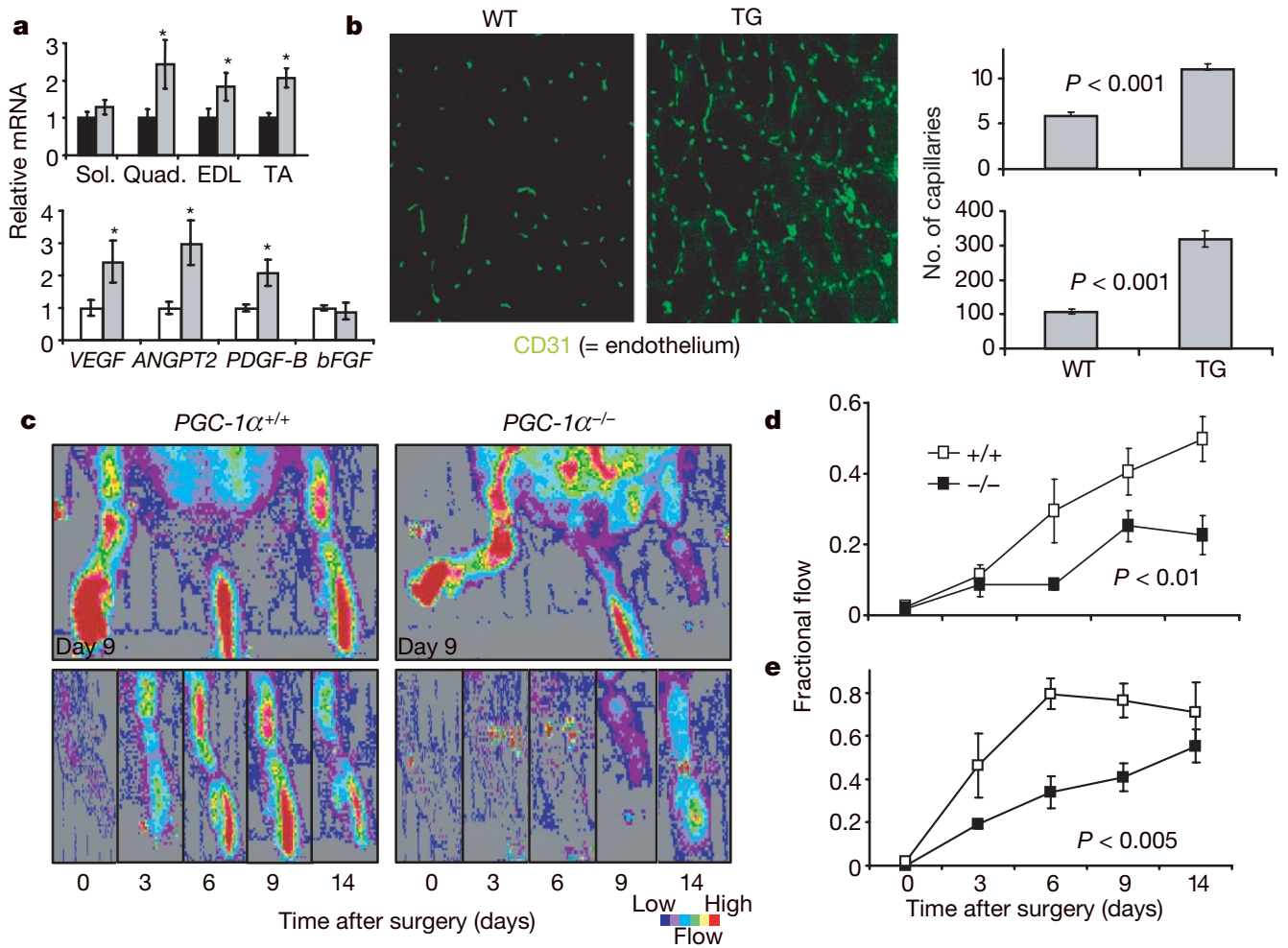

Figure 2 | PGC-1 $\alpha$ regulates angiogenesis and blood flow recovery after ischaemia in vivo. a, Top: expression of VEGF mRNA in wild-type (black bars) and muscle creatine kinase (MCK)-PGC- $1 \alpha$ transgenic (grey bars) animals. Bottom: expression of the indicated mRNAs in quadriceps from wild-type (white bars) and MCK-PGC- $1 \alpha$ transgenic (grey bars) animals. Sol., soleus; quad., quadriceps; EDL, extensor digitorum longus; TA, tibialis anterior. $n=5$ per group. $b$, Left: transverse sections of TA muscle from wild-type (WT) and transgenic (TG) animals stained for CD31. Right: quantification of capillaries per fibre (top) and per high-power field (bottom). $n=6$ high-power fields from three mice per group. c, d, $P G C$ $1 \alpha^{+/+}$and $P G C-1 \alpha^{-/-}$mice subjected to femoral artery ligation were subsequently monitored by infrared imaging. Sample images are shown in c, and quantification of blood-flow recovery is shown in $\mathrm{d}$. $n=8$ per group. e, Blood flow recovery after treating WT (filled squares) and MCK-PGC-1 $\alpha$ TG (open squares) mice as in c. $n=6$ per group. Error bars indicate s.e.m. Asterisk, $P<0.05$ compared with control. 
contrast with less than $40 \%$ in wild-type controls. Hence, under these conditions, PGC- $1 \alpha$ contributes significantly to the physiological response to hindlimb ischaemia. PGC- $1 \alpha$ does not seem to affect functional VEGF signalling in endothelial cells (Supplementary Figs 9 and 10), which is consistent with a primary role for PGC-1 $\alpha$ in skeletal muscle cells.

The regulation of VEGF in response to hypoxia is thought to be mediated primarily through the transcription factors HIF- $1 \alpha$ and HIF- $2 \alpha$ (ref. 9). However, the regulation of VEGF by PGC- $1 \alpha$ does not seem to involve this pathway. The hypoxic response element (HRE) that is targeted by HIF is well characterized and, when placed upstream of a luciferase reporter gene, can be activated by the addition of HIF- $1 \alpha$ or HIF- $2 \alpha$, or by hypoxia itself. However, the addition of PGC- $1 \alpha$ has no effect on this activity, even if tested in the presence of exogenous HIF- $1 \alpha$ or HIF- $2 \alpha$, or at reduced oxygen tension (Supplementary Fig. 11). The same is true if the HRE is tested in the context of a larger fragment of the VEGF promoter (Supplementary Fig. 12). Delivery of PGC-1 $\alpha$ to cells by means of adenovirus has no effect on HIF-1 $\alpha$ gene expression or protein stability, even though VEGF is robustly induced (Fig. 3a). HIF-1 $\alpha$ is also not altered in $P G C-1 \alpha^{-1-}$ cells (Supplementary Fig. 13). HIF- $1 \alpha$ and HIF- $2 \alpha$ form obligate heterodimers with the aryl hydrocarbon receptor nuclear translocator (ARNT) subunit of HIF, and in cells lacking ARNT there is no HIF activity ${ }^{10}$. However, when PGC- $1 \alpha$ is delivered by means of adenovirus to cells lacking ARNT, a fourfold induction of VEGF is still seen, equivalent to that seen in wild-type control cells (Fig. 3b). Hence, the induction of VEGF by PGC- $1 \alpha$ does not require the HIF pathway.

Similarly, the induction of $P G C-1 \alpha$ by the deprivation of nutrients and oxygen is apparently independent of HIF. The promoter region of $P G C-1 \alpha$, placed upstream of a luciferase reporter gene, is not affected by the addition of a constitutively active form of HIF- $1 \alpha$, whereas the promoter region of VEGF is strongly activated (Supplementary Fig. 14). A number of small molecules, including $\mathrm{CoCl}_{2}$,

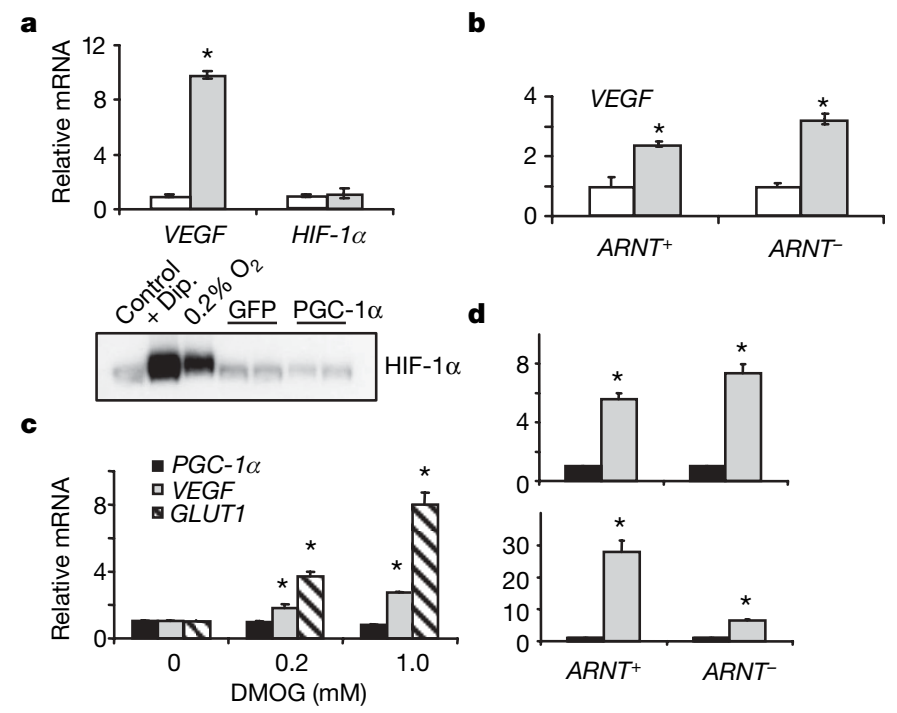

Figure 3 | PGC-1 $\alpha$ is induced by nutrient/oxygen deprivation and regulates VEGF independently of the HIF pathway. a, Primary skeletal muscle cells were infected with adenovirus expressing GFP (white bars) or PGC- $1 \alpha$ (grey bars). After $48 \mathrm{~h}, H I F-1 \alpha$ mRNA (top), and protein (bottom) was measured. HIF- $1 \alpha$ protein levels after treatment for $6 \mathrm{~h}$ with dipyridamole (dip.) or hypoxia $\left(0.2 \% \mathrm{O}_{2}\right)$ are shown as controls. ct, control. b, $A R N T^{-}$and $A R N T^{+}$cells were infected with adenovirus expressing GFP (white bars) or PGC- $1 \alpha$ (grey bars), and VEGF mRNA was measured. c, $\mathrm{C} 2 \mathrm{C} 12$ myotubes were treated with dimethyloxallyl glycine (DMOG) for $24 \mathrm{~h}$, and expression of the indicated mRNAs was measured. d, $A R N T^{-}$and $A R N T^{+}$cells were deprived of oxygen and nutrients for $3 \mathrm{~h}$ (grey bars), and expression of $P G C-1 \alpha$ mRNA (top) and VEGF mRNA (bottom) was measured. Black bars, controls. Error bars indicate s.e.m.; $n>3$ per group in all panels. Asterisk, $P<0.05$ compared with control. deferroxamine and dimethyloxallyl glycine, can activate the HIF pathway and induce VEGF, GLUT1 and other target genes; these molecules, however, have no effect on $P G C-1 \alpha$ expression (Fig. $3 \mathrm{c}$ and Supplementary Fig. 15). Finally, the induction of $P G C-1 \alpha$ by the deprivation of nutrients and oxygen is intact in cells lacking ARNT (Fig. 3d, top panel, and Supplementary Fig. 16), whereas the induction of canonical HIF targets is blunted (Fig. 3d, bottom panel, and Supplementary Fig. 17). Together, these data clearly show that PGC- $1 \alpha$ has a critical function in an angiogenic pathway that is independent of the canonical HIF pathway.

PGC- $1 \alpha$ is known to co-activate several transcription factors, including many members of the MEF2 (myocyte enhancer factor2), FOXO (forkhead transcription factor $\mathrm{O}$ ) and nuclear receptor families ${ }^{2}$. Plasmids expressing several of these transcription factors, with or without PGC- $1 \alpha$, were co-transfected into $10 \mathrm{~T} 1 / 2$ cells along with a reporter plasmid containing 2 kilobases of the VEGF promoter driving the luciferase gene. The addition of PGC- $1 \alpha$ to nuclear respiratory factor (NRF)-1 or NRF-2 (Fig. 4a), MEF2s or FOXO1 (data not shown) had no effect on luciferase activity. The addition of PGC- $1 \alpha$ to the orphan nuclear receptor ERR- $\alpha$, in contrast, led to an eightfold induction of luciferase activity (Fig. 4a). ERR- $\alpha$ is known to interact physically and functionally with PGC- $1 \alpha$ and is involved in the activation of programmes of fatty acid oxidation and oxidative phosphorylation $^{11-16}$. The induction of luciferase activity by PGC-1 $\alpha$ plus ERR- $\alpha$ did not require the integrity of the HIF-responsive element in the VEGF promoter (Supplementary Fig. 18). Instead, it required the integrity of two putative ERR- $\alpha$-binding sequences, both of which are perfectly conserved across vertebrate species (Supplementary Fig. 19). To examine the role of ERR- $\alpha$ in regulating endogenous VEGF, primary skeletal muscle cells were infected with adenovirus expressing ERR- $\alpha$. This led to a threefold induction of VEGF mRNA, compared with infection with a control virus expressing GFP alone (Fig. 4b). As a critical test for whether ERR- $\alpha$ is required for the PGC- $1 \alpha$-mediated induction of VEGF, primary mouse embryonic fibroblasts were prepared from $E R R-\alpha^{-I-}$ and wild-type animals, and the expression of VEGF was evaluated after infection with adenovirus expressing PGC- $\alpha$. In wild-type mouse embryonic fibroblasts, PGC- $1 \alpha$ induced VEGF expression sevenfold, compared with virus expressing GFP alone (Fig. 4c). In sharp contrast, the induction of VEGF by PGC- $1 \alpha$ was completely abrogated in $E R R-\alpha^{-1-}$ mouse embryonic fibroblasts. The induction of PDGF-B by PGC- $1 \alpha$ was also abrogated in these cells (Supplementary Fig. 20). Consistent with these findings is our observation that the expression of VEGF in ERR- $\alpha^{-1-}$ primary skeletal muscle cells was reduced by about $50 \%$ (Supplementary Fig. 21). Thus, PGC- $1 \alpha$ seems to stimulate VEGF expression at least in part by the coactivation of ERR- $\alpha$.

ERR- $\alpha$ recognizes the consensus DNA sequence AAGGTCA ${ }^{17}$. A search through the 25 kilobases surrounding the murine VEGF gene revealed the existence of 11 such sites, whereas only three or four would have been predicted by chance alone (Fig. 4d). Of these 11 sites, 6 are perfectly conserved between human, mouse and rat (red arrows in Fig. 4d); strikingly, 5 of them are clustered within regions of high homology in the first intron of the VEGF gene. A 1,200-basepair region encompassing these five sites was amplified by polymerase chain reaction and cloned upstream of the $S V 40$ promoter and a luciferase reporter gene. Co-transfection of $P G C-1 \alpha$ and $E R R-\alpha$ with this reporter plasmid led to a synergistic sevenfold induction of luciferase activity (Fig. 4 e, left half). By contrast, PGC- $1 \alpha$ and ERR- $\alpha$ had no effect on a control plasmid containing the $S V 40$ promoter alone (Fig. $4 \mathrm{e}$, right half). The induction of luciferase activity was dependent on the integrity of the conserved ERR- $\alpha$ sites (Supplementary Fig. 22). Chromatin immunoprecipitation assays revealed that PGC- $1 \alpha$ can occupy these ERR- $\alpha$ sites as well as the ERR- $\alpha$ sites found on the promoter (Fig. 4f). Thus, the first intron of the VEGF gene contains a putative enhancer region in which several conserved 
a
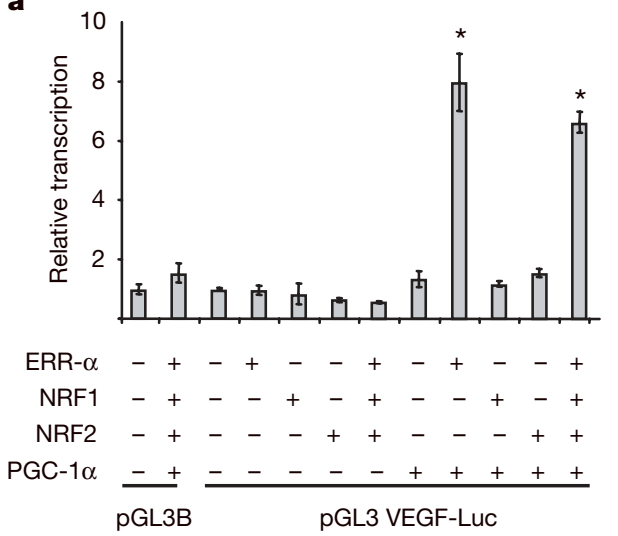

e

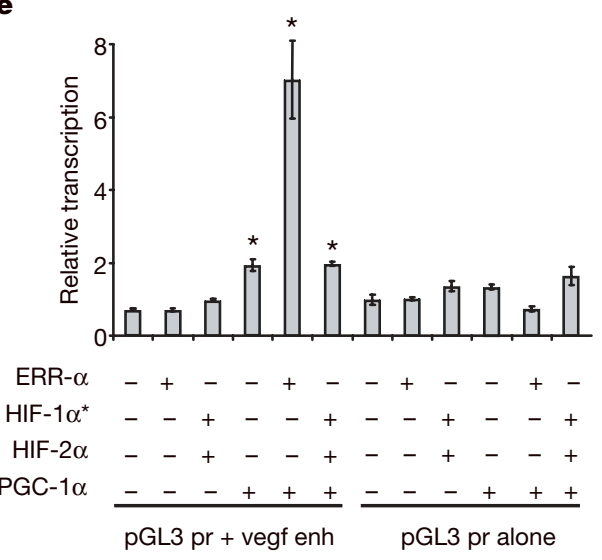

b

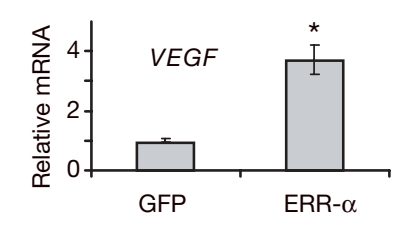

c

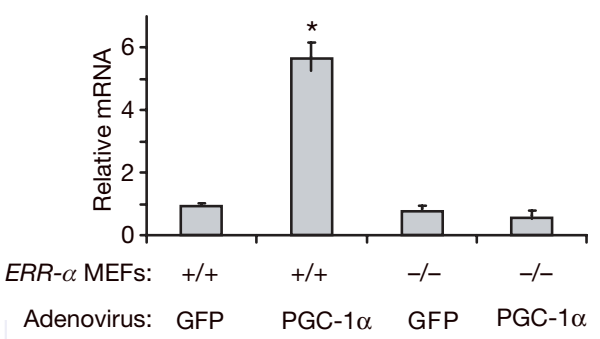

$\mathbf{g}$
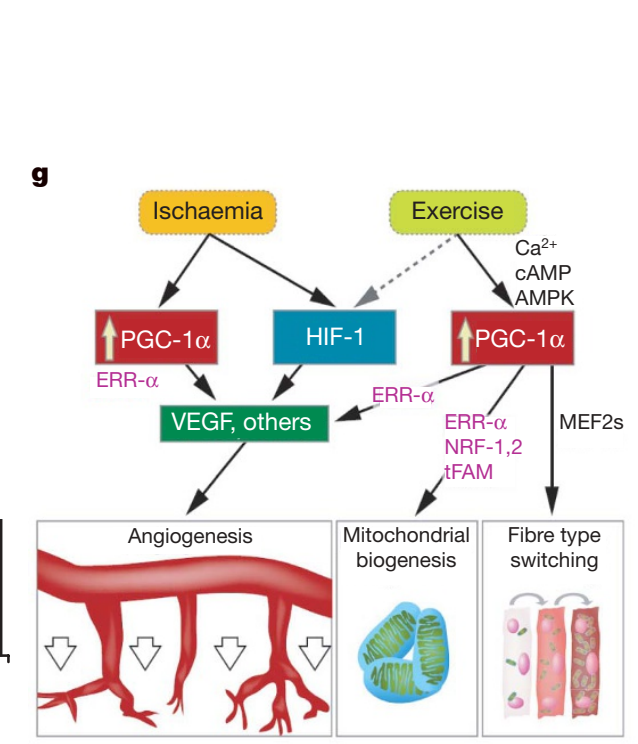

$\mathbf{f}$
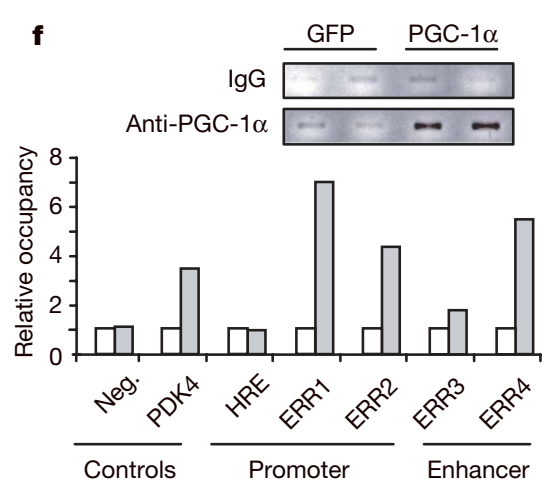
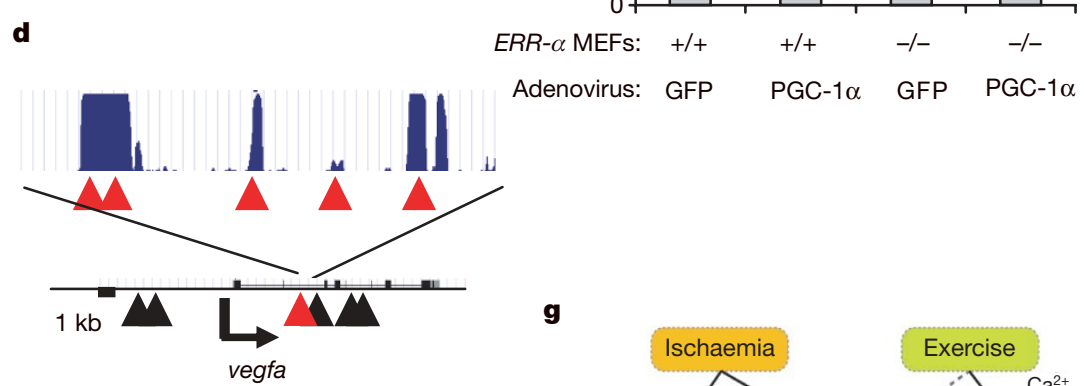

Figure 4 | PGC-1 $\alpha$ regulates expression of VEGF through coactivation of ERR- $\alpha$. a, luciferase (Luc) activity in cells transfected with VEGF or control promoter constructs, plus vectors expressing the indicated proteins. b, VEGF mRNA in primary skeletal myotubes infected with adenovirus expressing GFP or PGC- $1 \alpha . \mathrm{c}, E R R-\alpha^{+/+}$and $E R R-\alpha^{-1-}$ mouse embryonic fibroblasts evaluated as in b. d, Diagram of the murine vegfa gene region. Arrowheads indicate consensus ERR- $\alpha$ binding sites; red arrowheads indicate sites perfectly conserved between mammals. kb, kilobase. The histogram shows interspecies homology. See Supplementary Fig. 15 for details. e, Luciferase activity in cells transfected with the indicated plasmids, plus a reporter

construct containing the region depicted in d. pr, SV40 promoter; vegf enh, vegf enhancer. f, Chromatin immunoprecipitations in $\mathrm{C} 2 \mathrm{C} 12$ myotubes infected with the indicated adenovirus. White bars, IgG; grey bars, PGC- $1 \alpha$. ERR1-ERR4, putative ERR- $\alpha$-binding sites in the VEGF promoter and enhancer (see Supplementary Figs 13 and 15); PDK4, known site in the PDK4 promoter; HRE, hypoxic responsive element; neg., unrelated site. $\mathrm{g}$, Speculative model for the role of PGC- $1 \alpha$ in the regulation of angiogenesis during exercise and in response to ischaemia. AMPK, AMP-activated protein kinase; tFAM, mitochondrial transcription factor A. Error bars indicate s.e.m. Asterisk, $P<0.05$ compared with control.

ERR- $\alpha$-binding sites are recognized by ERR- $\alpha$ and coactivated by PGC- $1 \alpha$ to elicit the robust induction of VEGF transcription.

The data here show that PGC- $1 \alpha$ is a mediator of signalling in response to deprivation of nutrients and oxygen, and that it powerfully regulates $V E G F$ and other angiogenic factors to elicit neovascularization in vivo. The regulation of VEGF in response to hypoxia is thought to be mediated primarily through the well-known HIF factors ${ }^{9}$. Surprisingly, the novel PGC- $1 \alpha /$ ERR- $\alpha$ pathway described here is apparently independent of the HIF pathway. $P G C-1 \alpha^{-1-}$ mice are viable, suggesting that PGC- $1 \alpha$ is not essential in embryonic vascularization. Angiogenesis in the adult occurs in both physiological and pathological contexts ${ }^{1}$. The robust induction of vascularization by PGC- $1 \alpha$, and its critical function in the response to limb ischaemia, strongly implicate PGC- $1 \alpha$ in the angiogenic response to ischaemia, providing protection against further ischaemic insults (Fig. 4g). PGC- $1 \alpha$ is also robustly induced by exercise and mediates known responses to exercise such as fibre-type switching and mitochondrial biogenesis ${ }^{3,7}$. On the basis of these observations and the data presented here, we speculate that the PGC- $1 \alpha /$ ERR- $\alpha$ pathway also mediates exercise-induced neovascularization (Fig. 4g). This elegantly links the regulation of consumption of oxygen by mitochondria to the delivery of oxygen and nutrients by the vasculature. Angiogenesis is also crucial to tumour progression and

metastasis. The interface of metabolism with cancer progression has been the subject of renewed scrutiny in recent years ${ }^{18}$. It will be of great interest to elucidate the role of PGC- $1 \alpha$ and ERR- $\alpha$ in this interface, given the important function of these molecules in metabolic control.

Human clinical trials that examine the efficacy of VEGF delivery as therapy in various settings, including chronic limb ischaemia, have yielded disappointing results ${ }^{6,19,20}$. In large part this may be because the use of VEGF alone seems to lead to immature, leaky vessels $^{19}$. The generation of fully functional vessels requires the coordinated action of numerous signals, such as PDGF-BB and the angiopoetins ${ }^{6}$. One therapeutic approach to this problem may be to modulate a transcriptional regulator that coordinates these signals appropriately ${ }^{6,21}$. The PGC- $1 \alpha /$ ERR- $\alpha$ pathway provides such an opportunity.

\section{METHODS SUMMARY}

Nutrient and oxygen deprivation was induced by placing cells in Hanks balanced salt solution and $0.2 \%$ oxygen. Hindlimb ischaemia was induced by ligation of the femoral artery, as described ${ }^{8}$. Infections with adenovirus and transfections were performed as described ${ }^{22}$. One-way analysis of variance with repeated values was used to analyse data from femoral ligations. Two-tailed independent Student's $t$-tests were used to determine all other $P$ values. 
Full Methods and any associated references are available in the online version of the paper at www.nature.com/nature.

\section{Received 15 October; accepted 20 December 2007.}

1. Carmeliet, P. Angiogenesis in health and disease. Nature Med. 9, 653-660 (2003).

2. Lin, J., Handschin, C. \& Spiegelman, B. M. Metabolic control through the PGC-1 family of transcription coactivators. Cell Metab. 1, 361-370 (2005).

3. $\mathrm{Wu}$, Z. et al. Mechanisms controlling mitochondrial biogenesis and respiration through the thermogenic coactivator PGC-1. Cell 98, 115-124 (1999).

4. St-Pierre, J. et al. Bioenergetic analysis of peroxisome proliferator-activated receptor $\gamma$ coactivators $1 \alpha$ and $1 \beta$ (PGC-1 $\alpha$ and PGC-1 $\beta$ ) in muscle cells. J. Biol. Chem. 278, 26597-26603 (2003).

5. Semenza, G. L. Angiogenesis in ischemic and neoplastic disorders. Annu. Rev. Med. 54, 17-28 (2003).

6. Jain, R. K. Molecular regulation of vessel maturation. Nature Med. 9, 685-693 (2003).

7. Lin, J. et al. Transcriptional co-activator PGC-1 $\alpha$ drives the formation of slowtwitch muscle fibres. Nature 418, 797-801 (2002).

8. Couffinhal, T. et al. Mouse model of angiogenesis. Am. J. Pathol. 152, 1667-1679 (1998).

9. Ferrara, N., Gerber, H. P. \& LeCouter, J. The biology of VEGF and its receptors. Nature Med. 9, 669-676 (2003)

10. Wood, S. M., Gleadle, J. M., Pugh, C. W., Hankinson, O. \& Ratcliffe, P. J. The role of the aryl hydrocarbon receptor nuclear translocator (ARNT) in hypoxic induction of gene expression. Studies in ARNT-deficient cells. J. Biol. Chem. 271, 15117-15123 (1996).

11. Kelly, D. P. \& Scarpulla, R. C. Transcriptional regulatory circuits controlling mitochondrial biogenesis and function. Genes Dev. 18, 357-368 (2004).

12. Kressler, D., Schreiber, S. N., Knutti, D. \& Kralli, A. The PGC-1-related protein PERC is a selective coactivator of estrogen receptor alpha. J. Biol. Chem. 277, 13918-13925 (2002).

13. Schreiber, S. N., Knutti, D., Brogli, K., Uhlmann, T. \& Kralli, A. The transcriptional coactivator PGC-1 regulates the expression and activity of the orphan nuclear receptor estrogen-related receptor $\alpha$ (ERR $\alpha$ ). J. Biol. Chem. 278, 9013-9018 (2003).
14. Huss, J. M., Kopp, R. P. \& Kelly, D. P. Peroxisome proliferator-activated receptor coactivator-1 $\alpha$ (PGC-1 $\alpha$ ) coactivates the cardiac-enriched nuclear receptors estrogen-related receptor- $\alpha$ and $-\gamma$. Identification of novel leucine-rich interaction motif within PGC-1 $\alpha$. J. Biol. Chem. 277, 40265-40274 (2002).

15. Huss, J. M., Torra, I. P., Staels, B., Giguere, V. \& Kelly, D. P. Estrogen-related receptor $\alpha$ directs peroxisome proliferator-activated receptor $\alpha$ signaling in the transcriptional control of energy metabolism in cardiac and skeletal muscle. Mol. Cell. Biol. 24, 9079-9091 (2004)

16. Mootha, V. K. et al. Err $\alpha$ and Gabpa/b specify PGC-1 $\alpha$-dependent oxidative phosphorylation gene expression that is altered in diabetic muscle. Proc. Natl Acad. Sci. USA 101, 6570-6575 (2004).

17. Sladek, R., Bader, J. A. \& Giguere, V. The orphan nuclear receptor estrogen-related receptor $\alpha$ is a transcriptional regulator of the human medium-chain acyl coenzyme A dehydrogenase gene. Mol. Cell. Biol. 17, 5400-5409 (1997)

18. Shaw, R. J. Glucose metabolism and cancer. Curr. Opin. Cell Biol. 18, 598-608 (2006).

19. Carmeliet, P. VEGF gene therapy: stimulating angiogenesis or angioma-genesis? Nature Med. 6, 1102-1103 (2000).

20. Henry, T. D. et al. The VIVA trial: Vascular endothelial growth factor in Ischemia for Vascular Angiogenesis. Circulation 107, 1359-1365 (2003).

21. Pajusola, K. et al. Stabilized HIF-1 $\alpha$ is superior to VEGF for angiogenesis in skeletal muscle via adeno-associated virus gene transfer. FASEB J. 19, 1365-1367 (2005)

22. Arany, Z. et al. The transcriptional coactivator PGC-1 $\beta$ drives the formation of oxidative type IIX fibers in skeletal muscle. Cell Metab. 5, 35-46 (2007).

Supplementary Information is linked to the online version of the paper at www.nature.com/nature.

Acknowledgements We thank E. Smith for assistance with graphics. This work was supported by grants from the National Institutes of Health (Z.A. and B.M.S.), the Wenner-Gren Foundation (J.L.R.) and the Leduca Foundation (A.R. and B.M.S.).

Author Information Reprints and permissions information is available at www.nature.com/reprints. Correspondence and requests for materials should be addressed to Z.A. (zarany1@partners.org) or B.S.

(bruce_spiegelman@dfci.harvard.edu). 


\section{METHODS}

Animals. All animal experiments were performed in accordance with procedures approved by the Institutional Animal Care and Use Committee. Mice were maintained on a standard rodent chow diet with 12-h/12-h light/dark cycles. Hindlimb ischaemia was induced by ligation of the femoral artery, as described ${ }^{8}$. Blood flow was measured with a MoorLDI2 infrared scanner.

Cell culture, adenovirus infections, and transient transfections. Cells were maintained in DMEM medium in the presence of 10\% FBS. 10T1/2 fibroblasts, C2C12 myoblasts, $A R N T^{+}$and ARNT hepatoma cells (2712 and 2717) were from ATCC. Primary skeletal muscle cells were prepared as described ${ }^{23}$. Mouse embryo fibroblasts were prepared by homogenizing day-13.5 embryos from $E R R-\alpha$ heterozygous matings. Experiments were performed on cells that were passaged two to four times. Nutrient and oxygen deprivation was induced by placing cells in Hanks balanced salt solution and $0.2 \%$ oxygen. Transfections of $10 \mathrm{~T} 1 / 2$ cells were performed with Superfect (Qiagen) with $0.2 \mu \mathrm{g}$ of reporter plasmid plus $0.1 \mu \mathrm{g}$ of the indicated expression plasmids and $0.01 \mu \mathrm{g}$ of a control plasmid encoding Renilla luciferase. After $24 \mathrm{~h}$, cells were treated as indicated; after a further $24 \mathrm{~h}$, luciferase assays were performed (Promega Dual-Luciferase). Cells were infected with adenovirus at a multiplicity of infection of 10-30, and mRNA expression was measured $48 \mathrm{~h}$ later. The adenovirus expressing ERR- $\alpha$ was generated by Welgen. The adenovirus expressing PGC- $1 \alpha$ has been described.

Reagents and gene expression. All reagents were from Sigma unless otherwise indicated. VEGF enzyme-linked immunosorbent assay kit was from Calbiochem. Total RNA was prepared with the Trizol method (Invitrogen). mRNAs were quantified with quantitative real-time PCR by using SYBR green dye and an ABI 7300 machine. Genome analyses were performed with the University of California Santa Cruz genome browser (http://genome.ucsc.edu/). Chromatin immunoprecipitations were performed with the EZChip kit (Upstate) in accordance with the manufacturer's protocol. One-way analysis of variance with repeated values was used to analyse data from femoral ligations. Two-tailed independent Student's $t$-tests were used to determine all other $P$ values.

23. Megeney, L. A., Kablar, B., Garrett, K., Anderson, J. E. \& Rudnicki, M. A. MyoD is required for myogenic stem cell function in adult skeletal muscle. Genes Dev. 10, 1173-1183 (1996). 